\title{
Evaluation of the Use and Value of Nursing Care Plans in Nursing Practice at a Referral Hospital, Kigali, Rwanda: Nurses’ Perspectives
}

\author{
Gilbert Banamwana ${ }^{1}$, A. Smith Mandy ${ }^{2}$ \\ ${ }^{1}$ University of Rwanda, College of Medicine and Health Sciences, Rwanda \\ ${ }^{2}$ University of KwaZulu-Natal, School of Nursing \& Public Health, South Africa
}

\section{Background}

The use of nursing care plans within nursing process is geared to move toward a more systematic approach to human needs assessment. The nursing care plan will support practice modalities by meeting physical, psychological, social and spiritual needs of patients resulting in quality nursing care.

\section{Objectives}

The objectives of this study was to explore and describe the use of nursing care plans at a large teaching hospital in Rwanda.

\section{Methods}

A non-experimental quantitative cross-sectional survey design, descriptive in nature was adopted. A checklist was utilized to conduct a file review through a stratified random sample and fishbowl technique $(25 \%, \mathrm{n}=101)$. A self-report questionnaire was used to conduct an assessment of knowledge, attitudes, and perceptions of registered nurses $(n=28)$ in four units.

\section{Results}

This study showed that most nurses $(96 \%)$ felt that the use of nursing care plans increased the nurses' ability to provide high quality care; but only $16 \%$ of the audited files had evidence of a care plan. The main thrust of reported barriers to care plan utilization noted by registered nurses included a lack of training, inaccessibility to available evidence-based nursing practice, and heavy workload.

\section{Conclusion}

This study supports the need for creating increased awareness of the importance of nursing care plans and evidence based practice among registered nurses. Knowledge and training will increase the utilization of care plans by nurses' usage and they will add to the existing quality improvement in clinical practice in the hospital.

Key words: registered nurse, nursing care plans, quantitative survey 